\title{
LncROR Promotes Bladder Cancer Cell Proliferation, Migration, and Epithelial- Mesenchymal Transition
}

\author{
Yi Chen ${ }^{\mathrm{a}}$ Ya Peng ${ }^{\mathrm{b}}$ Zhipeng Xu $\mathrm{Xu}^{\mathrm{a}}$ Bo Ge ${ }^{\mathrm{a}}$ Xuebao Xiang ${ }^{\mathrm{a}}$ Tianyu Zhang ${ }^{\mathrm{a}} \mathrm{Li} \mathrm{GaO}^{\mathrm{a}}$ \\ Hailin Shia Chuang Wanga Jiefu Huanga \\ a Department of Urology, the Affiliated Hospital of Guilin Medical University, Guilin, ${ }^{\mathrm{b} C e n t r a l}$ Laboratory \\ of Basic Medical Science, Guangxi Medical University, Nanning, China
}

\section{Key Words}

Bladder cancer • IncRNA ROR • Proliferation • Migration • EMT

\begin{abstract}
Background: LncRNA ROR, a tumor oncogene associated with various human cancers, has been reported to be involved in regulating various cellular processes, such as proliferation, apoptosis and invasion through targeting multiple genes. However, the molecular biological function in bladder cancer has not been clearly elucidated. The aim of this study is to explore ROR expression levels and evaluated its function in bladder cancer. Methods: LncRNA ROR expression levels in the 36 pairs of bladder cancer tissues (and corresponding non-tumor tissues) and bladder cancer cells were assessed by qRT-PCR. MTT assay, colony formation assay, flow cytometric analysis, wound healing assay, cell transwell assays, attachment/detachment and western blotting were performed to assess the effects of ROR on proliferation, apoptosis, migration/invasion and epithelial-to-mesenchymal (EMT) phenotypes in BC cells in vitro. ZEB1 is target of ROR. Rescue assays were performed to further confirm that ROR contributes to the progression of BC cells through targeting ZEB1. Results: LncRNA ROR was up-regulated in bladder cancer tissues (compared to adjacent non-tumor tissues) and was almost overexpression in bladder cancer cells (compared with normal urothelial cell line SVHUC-1 cells). Increased IncRNA ROR expression significantly promoted tumor cells proliferation, inhibited cells apoptosis, facilitated cells metastasis and contributed to the formation of EMT phenotype. While down-regulated ROR could obviously inhibit cells proliferation, promote cells apoptosis, inhibit metastasis and reverse EMT to MET. ZEB1 was a target gene of ROR and was positive correlation with the level of ROR in cancer tissues. Conclusion: These results indicated that IncRNA ROR was associated with tumor progression in bladder cancer cells.

Y. Chen and Y. Peng contributed equally to this work. 


\section{Introduction}

Bladder cancer (BC) is one of the most common malignancies over the world, and its incidence and mortality have drawn great attention in the past decade [1]. Despite the development of treatments for patients with bladder cancer, the 5-year cancer-specific survival rate remains unsatisfied [2]. Lacking sophisticated understanding of pathogenetic mechanism is one of the most crucial reasons for high case fatality rate of bladder cancer. So, further revealing the pathogenetic mechanism of bladder cancer is indispensable for developing an effective diagnosis or treatment.

Long non-coding RNAs (lncRNAs), generally defined as transcripts longer than 200 nucleotides have captured significant attention in many biology processes [3-7]. Many lncRNAs, such as MALAT1, H19, and HOTAIR have been demonstrated as critical regulator of carcinogenesis and development of many cancers [8-12]. LncROR was first discovered in induced pluripotent stem cells (iPSCs). Loewer et al. identified lncRNA-ROR as a major regulator of pluripotency by examining lncRNA expression following fibroblast reprogramming into induced pluripotent stem cells (iPSCs) [13]. Recently, lncROR has been documented dysregulated in many cancers such as breast cancer, lung cancer, hepatocellul cancer, prostate cancer, gastric cancer and pancreatic cancer [14-23]. However, the function of IncROR in BC still remains unclear.

In the current study, we comprehensively investigated the biologic functions of lncROR in BC cells. Our results showed that lncROR was upregulated in BC tissues and investigated the effects of lncROR expression on bladder cancer cells in vitro. Moreover, further experiments indicated that the overexpression of lncROR could promote proliferation, inhibit apoptosis, facilitate migration and induce EMT of the bladder cancer cell lines, and such function was main dependent on targeting ZEB1 .

\section{Methods and Materials}

Patients and clinical samples collection

$\mathrm{BC}$ tissues $(\mathrm{n}=36)$ and pair-matched noncancerous tissues were obtained through tissue biopsy from patients diagnosed with BC at the Department of Urology, Affiliated Hospital of Guilin Medical University between March 2011 and September 2015. Informed consent was obtained from patients.

\section{Cell lines}

Bladder cancer UMUC3, SW780, 5637, T24 and normal urothelial cell line SVHUC-1 cells used in this study were purchased from the Tumor Cell Bank of the Chinese Academy of Medical Science (Shanghai, China). The UMUC3, T24 and SV-HUC-1 cells were cultured in Dulbecco's Modified Eagle Medium (Invitrogen, Carlsbad, CA, USA) plus $10 \%$ fetal bovine serum and ampicillin and streptomycin at $37^{\circ} \mathrm{C}$ in a humidified atmosphere of 95\% air and 5\% CO2. The 5637 cells and SW780 cells were cultured in RPMI-1640 Medium (Invitrogen, Carlsbad, CA, USA) plus $10 \%$ fetal bovine serum and ampicillin and streptomycin at $37{ }^{\circ} \mathrm{C}$ in a humidified atmosphere of $95 \%$ air and $5 \% \mathrm{CO} 2$.

Real-time quantitative reverse-transcription polymerase chain reaction ( $q R T-P C R$ )

Total RNA from tissues and cells was isolated with Trizol reagent (Invitrogen, CA, USA) according to the manufacturer's instructions. Reverse transcription was performed with PrimeScript RT reagent Kit (Takara, Japan) according to the manufacturer's protocol. qRT-PCR was performed with SYBR Prime Script RT-PCR Kits (Takara, Japan) based on the manufacturer's instructions. The ROR and ZEB1 level was calculated with the $2^{-\Delta \Delta C t \text { method }}$, which was normalized to GAPDH mRNA, respectively. All assays were performed in triplicate. The expression levels were relative to the fold change of the corresponding controls, which were defined as 1.0 .

Cell viability

Cell viability was assessed via 3-(4,5-dimethylthiazol-2-yl)-2, 5-diphenyl-trtrazolium bromide (MTT) assay. $5 \times 10^{3}$ cells/well were seeded in a 96-well flat-bottomed plate for $24 \mathrm{~h}$, then transfected 
with corresponding sh-RNA or pc-DNA3.1 and cultured in normal medium. At $0,24,48,72 \mathrm{~h}$ and $96 \mathrm{~h}$ after transfection, the MTT solution $(5 \mathrm{mg} / \mathrm{ml}, 20 \mu \mathrm{l})$ was added to each well. Following incubation for $4 \mathrm{~h}$, the media was removed and $100 \mu \mathrm{l}$ DMSO were added to each well. The relative number of surviving cells was assessed by measuring the optical density (O.D.) of cell lysates at $560 \mathrm{~nm}$. All assays were performed in triplicate.

\section{Colony formation assay}

Cells (500 cells/ well) were plated in 6-well plates and incubated in RPMI 1640 with 10\% FBS at $37^{\circ} \mathrm{C}$. Two weeks later, the cells were fixed and stained with $0.1 \%$ crystal violet. The number of visible colonies was counted manually.

\section{Flow cytometric analysis of apoptosis}

Apoptosis were performed using flow cytometric analyses with Annexin V: FITC Apoptosis Detection Kits (BD Biosciences, USA), according to the manufacturer's instructions. All samples were assayed in triplicate.

\section{Cell migration and invasion assays}

Cell migration and invasion were measured by transwell chamber ( 8 um pore size, Corning) and Matrigel invasion (Bection Dickinson), respectively. $48 \mathrm{~h}$ after transfection, cells in serum-free media were placed into the upper chamber without or with $10 \mathrm{ug} / \mathrm{ml}$ Matrigel. Media containing $10 \%$ FBS was added into the lower chamber. Following $48 \mathrm{~h}$ incubation, cells remaining in upper membrane were wiped off, while cells that migrated were fixed in methanol, stained with $0.1 \%$ crystal violet and counted under a microscope. Three independent experiments were carried out.

\section{Cell attachment and detachment assay}

For attachment assay, cells were seeded in 24 -well plates at $5 \times 10^{4}$ cells per well. Unattached cells were removed after $1 \mathrm{~h}$ incubation, and the attached cells were counted after trypsinization. The data were presented as a percentage of the attached cells compared to total cells. For cell detachment assay, after $24 \mathrm{~h}$ incubation, the cells were incubated with $0.05 \%$ trypsin for $3 \mathrm{~min}$ to detach the cells. Then, the culture medium was added to inactivate the trypsin and the detached cells were collected. The remaining cells were incubated with $0.25 \%$ trypsin to detach and counted. The data were presented as a percentage of the detached cells to total cells.

\section{Cell transfection}

PcDNA3.1/ROR (pcDNA3.1 as control), sh-ROR (shRNA as control) and pcDNA3.1/ZEB1 (pcDNA3.1 as control) were purchased from Applied Biological Materials (ABM, Canada). Transfections were performed using the Lipofectamine 2000 kit (Invitrogen) according to the manufacturer's instructions.

\section{Western bolt analysis and antibodies}

Total protein lysates were separated in $10 \%$ sodium dodecyl sulfate-polyacrylamide gel electrophoresis (SDS-PAGE), and were electrophoretically transferred to polyvinylidene difluoride membranes (Roche). Protein loading was estimated using mouse anti-GAPDH monoclonal antibody. The membranes were blotted with 10\% non-fat milk in TBST for $2 \mathrm{~h}$ at room temperature, washed and then probed with the rabbit antihuman E-cadherin (1: 2000 dilution), $\beta$-catenin (1: 2000 dilution), $\mathrm{N}$-cadherin (1: 2000 dilution), vimentin (1: 2000 dilution), ZEB1 (1: 2000 dilution), activated caspase-3 (1: 2000 dilution), total caspase-3 (1: 2000 dilution), activated caspase-9 (1: 2000 dilution), total caspase-9 (1: 2000 dilution), activated PARP (1: 2000 dilution), total PARP (1: 2000 dilution),and GAPDH (1: 3000 dilution), overnight at $4{ }^{\circ} \mathrm{C}$, followed by treatment with secondary antibody conjugated to horseradish peroxidase for $2 \mathrm{~h}$ at room temperature. The proteins were detected using an enhanced chemiluminescence system and exposed to $\mathrm{x}$-ray film. All antibodies were purchased from Abcam (USA).

\section{Statistical analysis}

Data are shown as the means \pm standard error of at least three independent experiments. The SPSS 17.0 software (SPSS Inc., Chicago, IL, USA) was used for statistical analysis. Two group comparisons 
were performed with a Student t test. Multiple group comparisons were analyzed with one-way ANOVA. Statistically significant positive correlation between ROR and ZEB1 expression levels in 36 cases of bladder cancer tissues was analyzed by Spearman's correlation analysis. Statistically significant positive correlation between ROR and ZEB1 expression levels in 36 cases of bladder cancer tissues was analyzed by Spearman's correlation analysis. All tests performed were two-sided. $\mathrm{P}<0.05$ was considered statistically significant.

\section{Results}

LncROR is up-regulated in bladder cancer tissues and cancer cells

To investigate the function of IncROR in BC, we first examined the expression of lncROR in 36 pairs of $\mathrm{BC}$ tissues and pair-matched histologically normal tissues by quantitative reverse-transcription polymerase chain reaction (qRT-PCR). Compared with matched noncancerous tissue (Fig. 1A), lncROR levels were increased in cancerous tissues $(p<0.01)$. Next, we examined the expression of IncROR in four BC cell lines UMUC3, SW780, 5637, T24 and normal urothelial cell line SVHUC-1 cells. As presented in Fig. 1B, obviously increased expression of lncROR was observed in 5637 cell lines, and the level of IncROR in UMUC3 was nearly as low as that in normal urothelial cell line SVHUC-1. These results indicated that lncROR might be involved in the progression of the BC.

Lower Inc-ROR expression correlates with poor prognosis in patients with BC

As shown in Table1, lnc-ROR expression was found to be correlated with tumor range, metastasis, lymphatic Nodes and pathological stage $(P=0.000,0.000,0.001$ and 0.000 , respectively). Proportional hazards method analysis revealed that low lnc-ROR expression ( $\mathrm{P}$ $=0.011$ ) was an adverse prognostic factor, in addition to the independent prognostic impact of Lymph Nodes (Table 2, $\mathrm{P}=0.030$ ). These results suggest that lnc-ROR plays a critical role in $\mathrm{BC}$ and may be considered as a specific biomarker of poor prognosis.

LncROR contribute to the proliferation of bladder cancer cells

To examine the biological functions of IncROR in BC cells, we overexpressed or downregulated lncROR in UMUC3 or 5637cells through transfected with lncROR expression vector pcDNA3.1/ IncROR or IncROR-specific shRNA, respectively, using the empty vector or control shRNA as a negative control (NC). Satisfactory transfection efficiency was obtained at $48 \mathrm{~h}$ post-transfection (Fig. 2A). Results from MTT assays showed increased proliferation

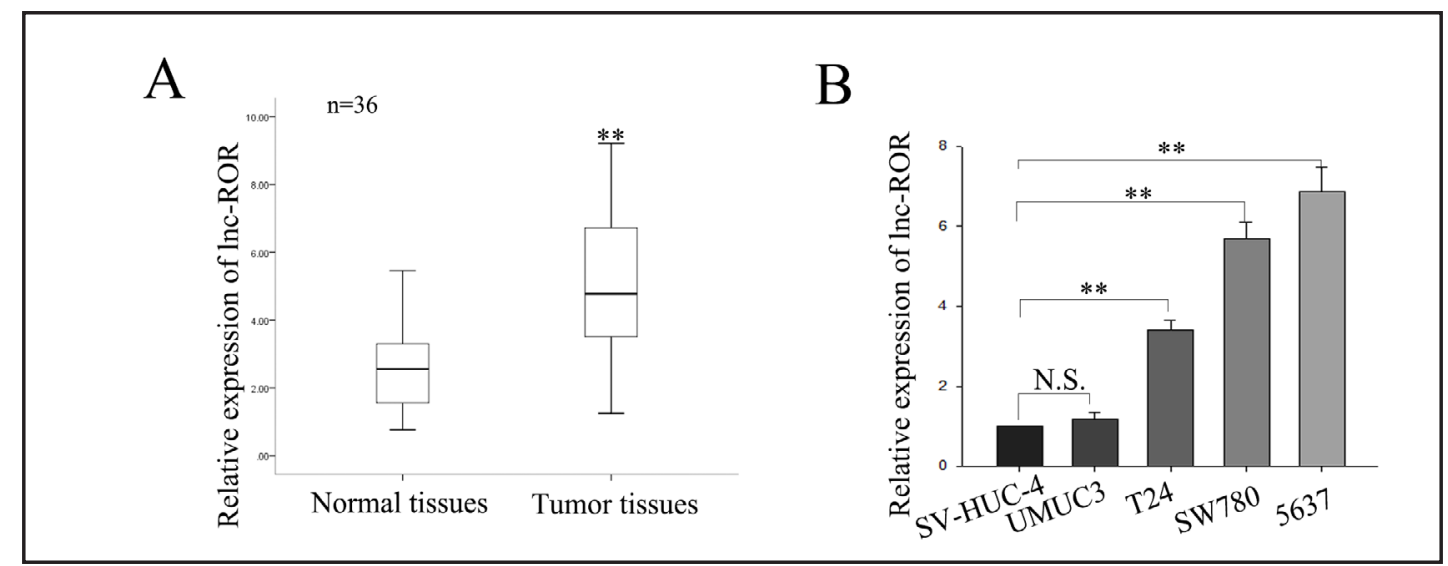

Fig. 1. IncRNA ROR is up-regulated in bladder cancer tissues and cancer cells. A. Differences in ROR expression levels between bladder cancer tissues and pair-matched noncancerous tissues. The expression of ROR was normalized to that of GADPH. Statistical differences between samples were analyzed with paired samples t-test $(n=36, p<0.01)$. B. Expression level of ROR in four BC cell lines UMUC3, SW780, 5637, T24 and normal urothelial cell line SVHUC-1 cells. Data are presented as mean \pm standard error based on at least three independent experiments. ${ }^{*} \mathrm{p}<0.05,{ }^{* *} \mathrm{p}<0.01$. 
Table 1. Correlation between lncROR Expression and Clinical Features. $(n=36)$. Low/ high by the sample median. Pearson $\chi^{2}$ test. $\mathrm{P}<0.05$ was considered statistically significant

\begin{tabular}{|c|c|c|c|}
\hline Variable & $\begin{array}{l}\text { Lnc-ROR Expression } \\
\text { low }\end{array}$ & high & P-value \\
\hline \multicolumn{4}{|l|}{ Age } \\
\hline$<60$ & 4 & 8 & \multirow{3}{*}{0.289} \\
\hline$\geq 60$ & 14 & 10 & \\
\hline \multicolumn{3}{|l|}{ Gender } & \\
\hline Male & 16 & 15 & \multirow{3}{*}{1.000} \\
\hline Female & 2 & 3 & \\
\hline \multicolumn{3}{|l|}{ Smoking } & \\
\hline No smoking & 5 & 3 & \multirow{2}{*}{0.691} \\
\hline Smoking & 13 & 15 & \\
\hline \multicolumn{4}{|c|}{ Tumor Range } \\
\hline T1-T3 & 18 & 4 & \multirow{2}{*}{0.000} \\
\hline$\geq \mathrm{T} 4$ & 0 & 14 & \\
\hline \multirow{2}{*}{\multicolumn{4}{|c|}{ Metastasis }} \\
\hline & 18 & 2 & \multirow{2}{*}{0.000} \\
\hline positive & 0 & 16 & \\
\hline \multicolumn{4}{|l|}{ Lymph Nodes } \\
\hline negative & 11 & 1 & \multirow{2}{*}{0.001} \\
\hline positive & 7 & 17 & \\
\hline \multicolumn{4}{|c|}{ Pathological Stage } \\
\hline$<\mathrm{IV}$ & 17 & 2 & \multirow{2}{*}{0.000} \\
\hline$\geq \mathrm{IV}$ & 1 & 16 & \\
\hline
\end{tabular}

Table 2. Multivariate analysis of prognostic parameters inpatients with bladder cancer by Cox regression analysis. Proportional hazards method analysis showed a positive, independent prognostic importance of lncROR expression $(\mathrm{P}=0.011)$, in addition to the independent rognostic impact of Lymph Nodes $(\mathrm{P}=0.030)$. $\mathrm{P}<0.05$ was considered statistically significant

\begin{tabular}{|c|c|c|}
\hline Variable & Category & P-value \\
\hline Age & $\begin{array}{l}<60 \\
\geq 60\end{array}$ & 0.207 \\
\hline Gender & $\begin{array}{l}\text { Male } \\
\text { Female }\end{array}$ & 0.741 \\
\hline Smoking & $\begin{array}{l}\text { No smoking } \\
\text { Smoking }\end{array}$ & 0.328 \\
\hline Tumor Range & $\begin{array}{l}\text { T1 } \\
\text { T2 } \\
\text { T3 } \\
\text { T4 }\end{array}$ & 0.449 \\
\hline Metastasis & $\begin{array}{l}\text { negative } \\
\text { positive }\end{array}$ & 0.884 \\
\hline Lymph Nodes & $\begin{array}{l}\text { negative } \\
\text { positive }\end{array}$ & $0.030^{*}$ \\
\hline Pathological Stage & $\begin{array}{l}<\mathrm{IV} \\
\geq \mathrm{IV}\end{array}$ & 0.215 \\
\hline Lnc-ROR expression & $\begin{array}{l}\text { Low } \\
\text { High }\end{array}$ & $0.011^{*}$ \\
\hline
\end{tabular}

ability of UMUC3 cells transfected with IncROR compared with NC-transfected cells. On the contrary, weakened proliferation ability was observed in IncROR-downregulated 5637 cells compared with NC-transfected cells ( $\mathrm{p}<0.01 ;$ Fig. $2 \mathrm{~B})$. Additionally, colony formation assays revealed higher proliferation of UMUC3 cells transfected with lncROR compared with NCtransfected cells $(\mathrm{p}<0.01$; Fig. $2 \mathrm{C})$. In contrast, suppression of proliferation was observed in IncROR-downregulated 5637 cells compared with NC-transfected cells $(\mathrm{p}<0.01$; Fig. 2C). Furthermore, we used flow cytometry analyses to assess the effect of lncROR on apoptosis. Compared with negative controls, forced expression of IncROR caused a decrease in apoptosis in UMUC3 cells ( $<<0.01$, Fig. 2D). Likewise, downregulation of IncROR resulted in increased apoptosis in 5637 cells compared with the NC group (Fig. 2D). Next, we measured the levels of apoptosis-related proteins (activated caspase-3, total caspase-3, activated caspase-9, total caspase-9, activated PARP and total PARP proteins). Overexpression of lncROR increased the levels of activated caspase-3, -9 , and PRAP in UMUC3 cells while downregulation of lncROR in 5637 cells decreased it; however, total proteins levels remained unchanged (Fig. 2E). Therefore, IncROR might activate the caspase-3-dependent apoptosis pathway. Taken together, these data suggest that lncROR overexpression enhanced their proliferation while reducing apoptosis of $\mathrm{BC}$ cells.

LncROR promotes migratory and invasive capacities and enhances the epithelial-tomesenchymal transition of bladder cancer cells

Metastasis/invasion is one of hallmarks of cancer. To investigate the function of ROR in regulating cell motility, we performed the migration /invasion assays and wound healing 


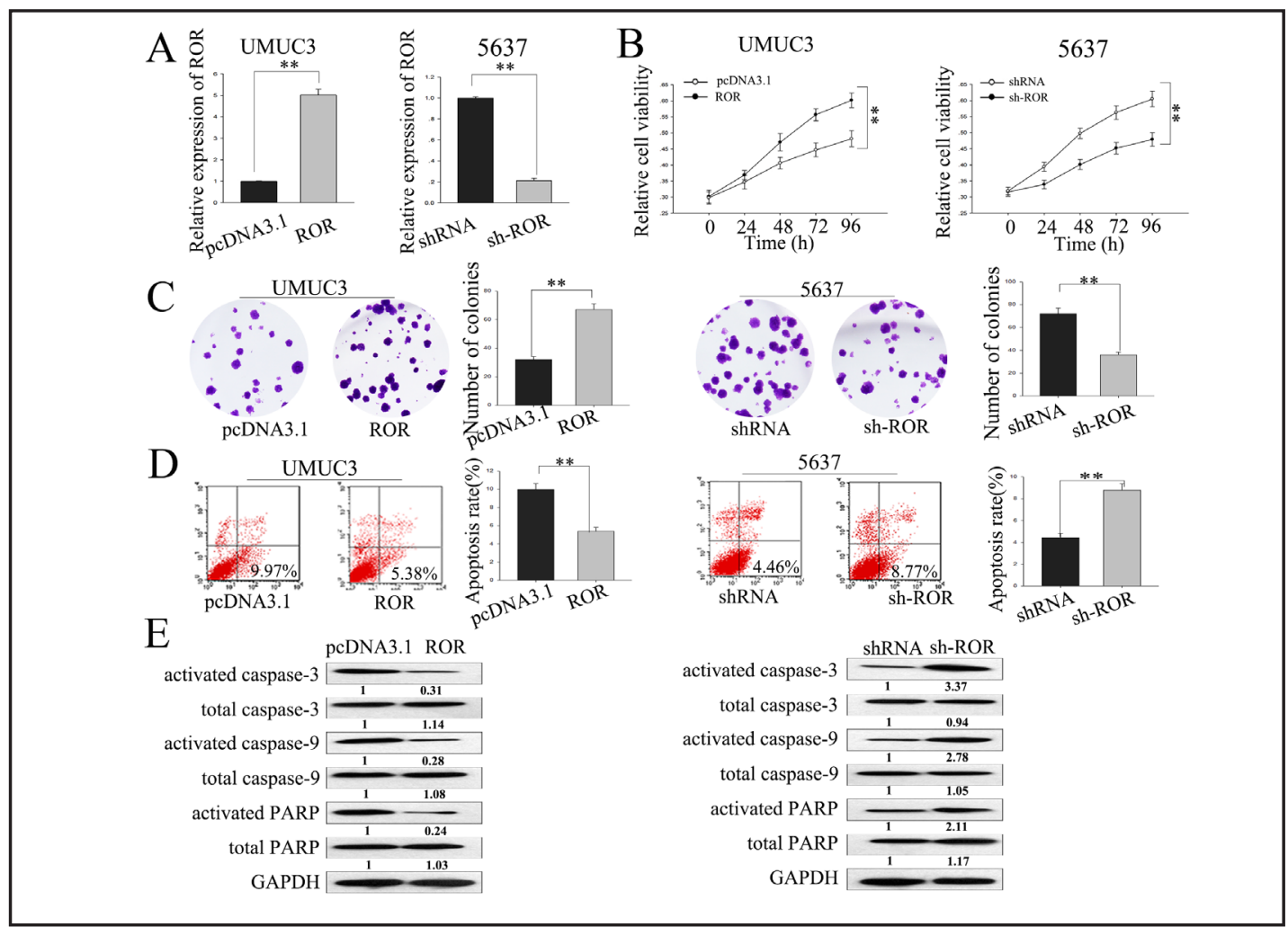

Fig. 2. IncRNA ROR contribute to the proliferation of bladder cancer cells. A. qRT-PCR assay was performed to examine the expression of ROR $48 \mathrm{~h}$ in UMUC3 cells transfection with ROR expression vector pcDNA3.1/ ROR (or control) and 5637 cells with ROR-specific shRNA (or shRNA control). B-C. MTT and colony formation assays on UMUC 3 cells transfected with ROR, and 5637 cells transfected with sh- ROR. D. Flow cytometry of UMUC3 cells transfected with ROR, and 5637 cells transfected with sh-ROR. E. Western blot of apoptosis related proteins (activated caspase-3, total caspas-3, activated caspase-9, total caspase-9, activated PARP and total PARP). Error bars represent the mean \pm SEM of at least three independent experiments. ${ }^{*} p<0.05$, ${ }^{* *} \mathrm{p}<0.01$ vs. control group.

assays in BC cells. As is shown in Fig. 3A, overexpression of ROR enhanced the wound healing ability of UMUC3 cells, and deletion of ROR weakened the healing ability of 5637 cells. Additionally, the migration assay present that overexpression of ROR could facilitate the migration/invasion capacity of UMUC3 cells, and down-regulated ROR in 5637 cells could significant inhibit cells migration/invasion capability (Fig. 3B). Consistent with this, highlevel of ROR promoted cell detachment and attachment in UMUC3 cells and down-expression of ROR inhibited cell detachment and attachment in 5637 cells (Fig. 3C). Furthermore, cell morphology and western blot assays revealed that high level of ROR in UMUC3 cells could induce formation of EMT and down-expression of ROR reverse the EMT to MET (Fig. 3D). These data together demonstrated that ROR was involved in the progression of BC cells.

\section{ZEB1 was target of ROR and mediated the function of ROR in BC cells}

It has been identified that ZEB1 was a target of ROR [16]. To detect whether ROR exerts its function in BC cells is dependent on ZEB1. We examined the level of ZEB1 in response to the level of ROR. As is shown in Fig. 4A and B, forced expression of ROR could increase the protein level of ZEB1 and depletion of ROR decreased the level of ZEB1 both in mRNA and protein level. Then, rescue assays were applied to further illuminate that the function of ROR was dependent on ZEB1. MTT assays and colony formation assays revealed that the proliferation ability of 5637 cells was rescued when co-transfected with sh-ROR and ZEB1 (Fig. 4C and D). Flow cytometry analyses showed that the pro-apoptosis function sh-ROR in 


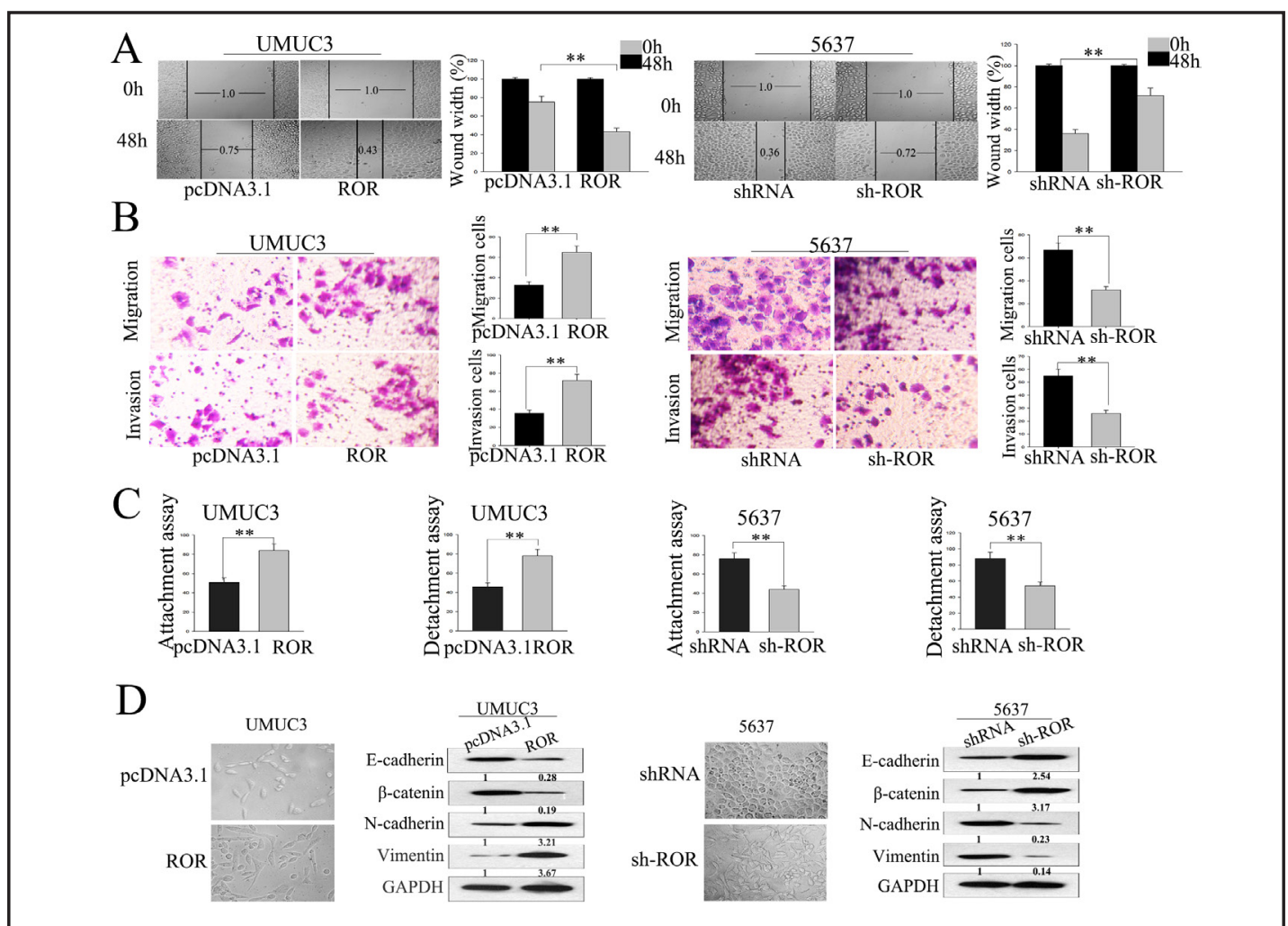

Fig. 3. IncRNA ROR facilitates the migration abilities. A-C., Wound healing, metastasis, and attachment/ detachment assays were utilized to detect the migratory capability of BC cells transfected with ZBE1 or shROR. D. Cell morphology and western blot of epithelial markers (E-cadherin, $\beta$-catenin) and mesenchymal markers (N-cadherin, vimentin). Error bars represent the mean \pm SEM of at least three independent experiments. ${ }^{*} \mathrm{p}<0.05,{ }^{* *} \mathrm{p}<0.01$ vs. control group.

5637 cells was rescued when introduction of ZEB1 (Fig. 4E). Additionally, as shown in Fig. $5 \mathrm{~A}-\mathrm{C}$ wound healing assays, migration/invasion assays and attachment/detachment assays present that anti-metastasis function of sh-ROR could be partially rescued by introduction of ZEB1. Furthermore, ZEB1 could reverse the MET induced by sh-ROR to EMT (Fig. 5D). These data indicates that ROR exerts its function in BC cells is dependent on ZEB1.

ZEB1 was up-regulated in bladder cancer tissues and was positive correlation with the level of IncRNA ROR

To better investigate the correlations between ROR and ZEB1 in BC, we performed qRTPCR to detect the expression of ZEB1 in 36 tumor tissue samples. The relative level of ZEB1 expression in the tumor tissue samples was significantly higher than that in pair-matched histologically normal tissues ( $\mathrm{p}<0.01$, Fig. $6 \mathrm{~A}$ ). Moreover, there was a significant positive correlation between ZEB1 expression level and ROR expression level (2-tailed Spearman's correlation, $r=0.713$, p $<0.01$; Fig. 6B). Additionally, the level of lncROR in metastasis cancer tissues was higher than that in non-metastasis cancer tissues (Fig. 6C). Consistent, in metastasis cancer tissues, the expression of ZEB1 both in mRNA and protein level was higher than that in non-metastasis cancer tissues (Fig. 6D). Collectively, these findings indicated that lnc-ROR/ZEB1signaling pathway was involved in the proliferation and metastasis of BC.

\section{Discussion}

Accumulating evidence has shown that ncRNAs play a pivotal role in tumorigenesis [2426]. Currently, the participation of IncRNAs in a wide repertoire of biological processes has KARGER 


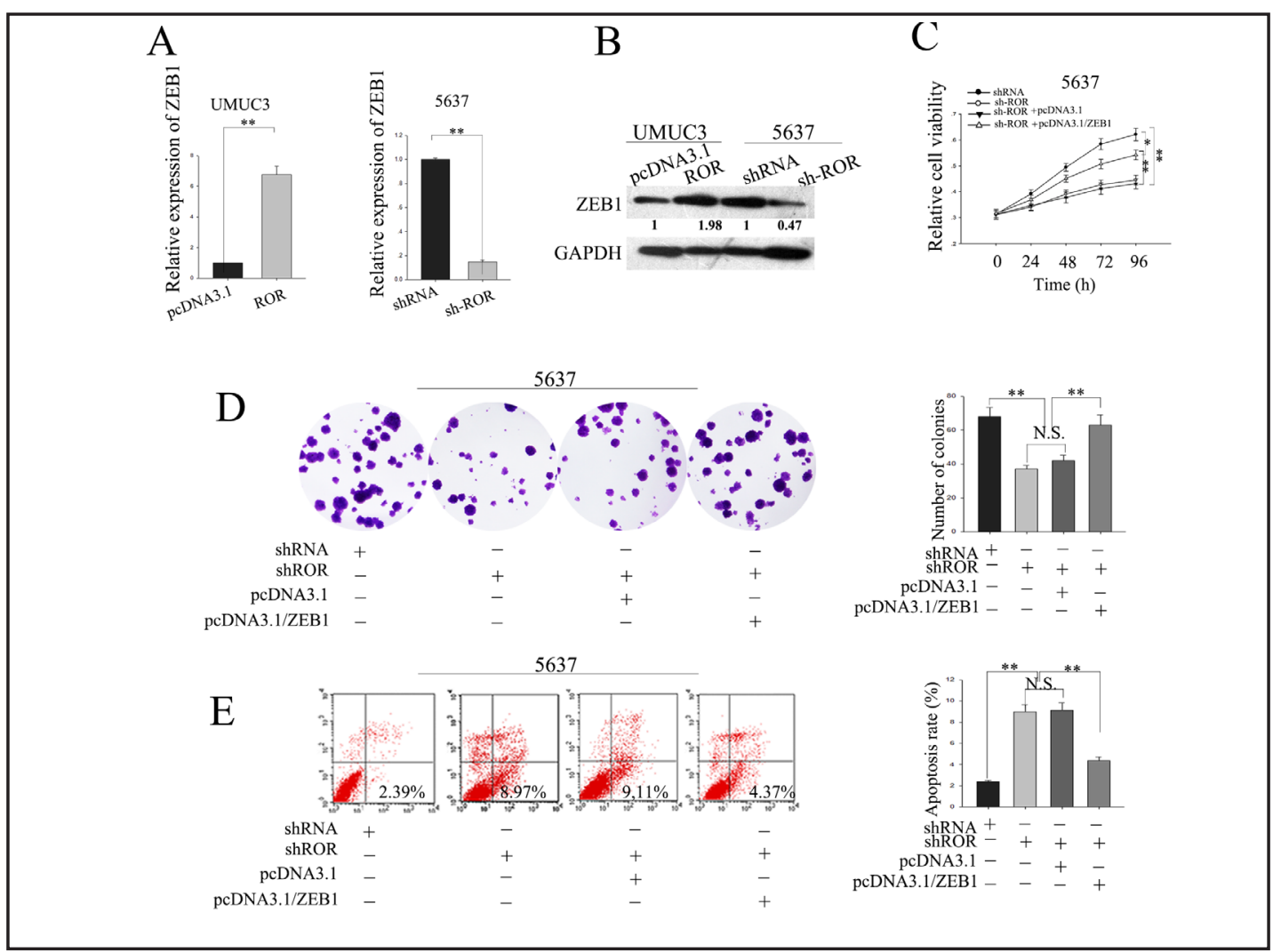

Fig. 4. ZEB1 was target of ROR and contribute to the proliferation of BC cells. A-B. qRT-PCR and western blot assays were utilized to detect the level of ZEB1 in response to the level of ROR both in mRNA and protein level. C-D. MTT and colony formation assays on 5637 cells co-transfected with sh- ROR and ZEB1. E. Flow cytometry of 5637 cells co-transfected with sh-ROR and ZEB1. Error bars represent the mean \pm SEM of at least three independent experiments. ${ }^{*} \mathrm{p}<0.05,{ }^{* *} \mathrm{p}<0.01$ vs. control group.

been a topic of intense research, and dysregulation of IncRNAs has indicated a vital function of these molecules in cancer pathogenesis and provided novel insights into therapeutics against cancers $[27,28]$. Despite these advances, the roles of lncRNAs in the carcinogenesis of $\mathrm{BC}$ are far from fully elucidated. Investigating the molecular mechanism by which lncRNAs function in $\mathrm{BC}$ might facilitate the exploitation of novel therapeutic targets. Besides, EMT, a biological process in which cancer cells lose their epithelial polarity and undergo transition into a mesenchymal phenotype, plays a key role in cancer cell malignant transformation. The emergence of EMT contributes to the malignancy of BC. Therefore, investigating the mechanism underlying the generation of $\mathrm{BC}$ and the EMT phenotype in $\mathrm{BC}$ is especially important for the development of novel treatment methods and improving patient survival rate.

The long non-coding RNAs (lncRNAs) are RNA molecules over $200 \mathrm{nt}$ in length with little protein-coding potentiall [29]. LncRNAs have been documented to regulate gene expression by a variety of mechanisms, for example, lncRNA H19 regulates glioma angiogenesis and the biological behavior of glioma-associated endothelial cells by targeting microRNA-29a, IncRNA ANRIL promotes the metastasis of thyroid cancer cells via regulating TGF-beta/ Smad signaling patheway, and IncRNA DUXAP8 promotes gastric cancer cell proliferation and migration via epigenetically silencing PLEKHO1 expression [30-32].

LncROR was first discovered in induced pluripotent stem cells (iPSCs), where it was regulated by the key pluripotency factors Oct4, Sox2 and Nanog. LncROR had a key role in the maintenance of iPSCs and embryonic stem cells (ESCs) by preventing the activation of cellular stress pathways, including the p53 response [13]. Subsequent studies unveiled that 


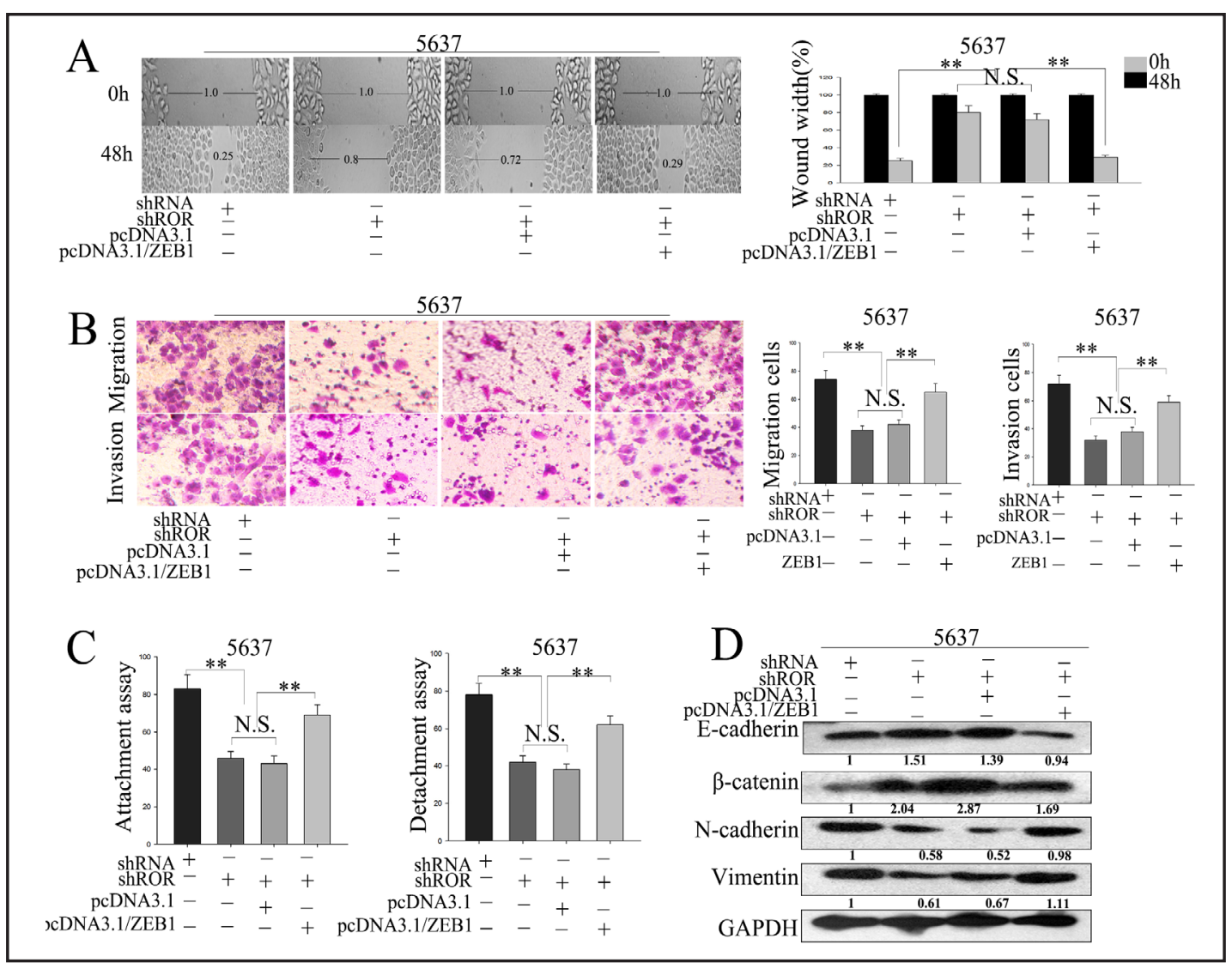

Fig. 5. IncRNA ROR facilitates the migration dependent on ZEB1 and induce EMT. A-C. Wound healing, metastasis and attachment/detachment assays were utilized to detect the migratory capability of BC cells co-transfected with sh-ROR and ZEB1. D. Western blot of epithelial markers (E-cadherin, $\beta$-catenin) and mesenchymal markers ( $\mathrm{N}$-cadherin, vimentin). Error bars represent the mean \pm SEM of at least three independent experiments. ${ }^{*} \mathrm{p}<0.05,{ }^{* *} \mathrm{p}<0.01$ vs. control group.

lincROR could act as a p53 repressor in response to DNA damage [33]. Moreover, it has been demonstrated that IncROR may function as a molecular sponge for mir-145 to upregulate the mir-145 targets, such as OCT4, SOX2 and Nanog expression [34]. Despite the implications of lncROR in the regulation of a variety of transcription factors and physiological processes, the role of lncROR in BC metastasis and EMT remains unexplored. Our results confirmed the oncogenic role of lncROR in BC, and identified ZEB1 as potential target gene of lncROR. Overexpression of ZEB1 increased the migration and invasion capacity and reversed MET to EMT in 5637 cells transfected with sh-ROR, indicating that ZEB1 was a potential target gene of lncROR, mediated the function exert of lncROR in BC cells.

In conclusion, our study was the first to investigate the role of lncROR in BC. The results indicated that lncROR was a novel potential oncogene in $\mathrm{BC}$, and was up-regulated in BC tissues. LncROR can promote proliferation, inhibit apoptosis, facilitate cell migration/ invasion and induce EMT. ZEB1 was a potential target gene of IncROR, and mediated the function exert of IncROR in BC cells, implicating the potential application of ROR in the prognosis and treatment of $\mathrm{BC}$.

Despite we revealed that the IncROR/ZEB1 signal pathway involves in regulating the progression of BC cells, the current research is still flawed. The main limitation of the present study is the exact regulatory mechanism between lncROR and ZEB1. Mechanisms of IncRNA regulating gene expression are mainly at three different levels: the transcriptional level, the post-transcriptional level and the epigenetic regulation level [35]. It has been reported that lncROR could function as competing endogenous RNA, therefore release the miRNA- 


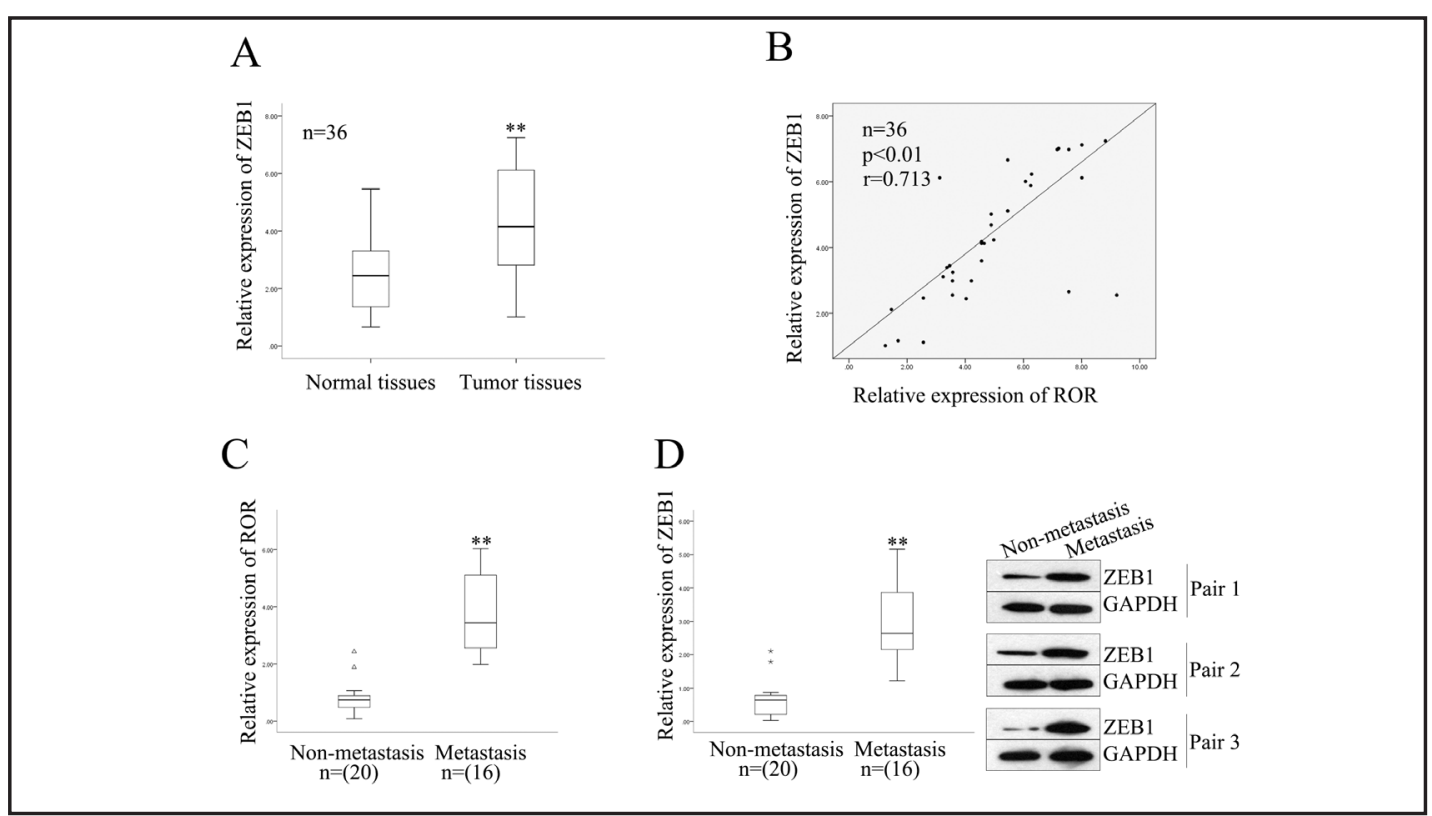

Fig. 6. ZEB1 was up-regulated in bladder cancer cells and was positive correlation with the level of lncRNA ROR. A. The expression of ZEB1 in 36 paired samples of bladder cancer tissues and pair-matched noncancerous tissues $(\mathrm{p}<0.01)$, B. showing positive correlation between ZEB1 expression and ROR expression in 36 $\mathrm{BC}$ tissues (2-tailed Spearman's correlation, $\mathrm{r}=0.779, \mathrm{p}<0.01$ ). C. level of lncROR in metastasis cancer tissues was higher than that in non-metastasis cancer tissues. D. the expression of ZEB1 in metastasis cancer tissues and non-metastasis tissues (left), the expression level of ZEB1 proteins in three pairs of non-metastasis and metastasis tissues (right). ${ }^{*} \mathrm{p}<0.05,{ }^{* *} \mathrm{p}<0.01$ vs. control group

combined target genes [36-38]. Moreover, lncROR has also been documented as a necessary decoy oncoRNA that plays an important regulatory role in tumorigenesis and represents a novel style of histone modification [39]. In addition to these reported regulating mechanisms of IncROR, another regulating mechanism of most well-defined lncRNAs, such as HOTAIR, IncRNA-p21, ANRIL, and MALAT-1, sharing a common functionality in the formation of RNAprotein complexes with chromatin regulatory factors [40]. In the future, we will focus on the regulating mechanism between lncROR and ZEB1. We will first knockdown Dicer enzymekey molecular in the process of miRNA maturation, to study whether miRNAs involves in lncROR-mediated function in BC cells. If knockdown Dicer enzyme, the function of lncROR vanishes or significantly decreases, we speculate that lncROR may function as a ceRNA in regulating ZEB1. If knockdown Dicer enzyme, the function of lncROR still exists, we will exam the methylation status of ZEB1 promoter or the relationship between IncROR and RNAprotein complexes with chromatin regulatory factors like PRC2 to study whether lncROR regulating ZEB1 through other pathways. In addition, in the future, we will also investigate the relationship of IncROR function and chemoresistant in BC cells, since chemotherapy is critical in treating BC patients.

In summary, the present study revealed that IncROR contributes to the progression of BC cells through targeting ZEB1. However, the regulatory mechanism between lncROR and ZEB1 remains uncovered. Therefore, in the future, we will further study the regulatory mechanism between IncROR and ZEB1. Additionally, we will also investigate the role of lncROR in regulating BC cells chemoresistant. Our ultimate goal is to provide a new train of thought for developing more efficient therapeutic agents.

\section{Acknowledgements}

This study was sponsored by Natural Science Foundation of Guangxi Province of China (2013gxnsfaao19223), Innovation Project of Guangxi Graduate Education (No. 


\section{Cellular Physiology Cell Physiol Biochem 2017;41:2399-2410 \\ \begin{tabular}{l|l} 
DOI: 10.1159/000475910 & $\begin{array}{l}\text { O 2017 The Author(s). Published by S. Karger AG, Basel } \\
\text { www.karger.com/cpb }\end{array}$
\end{tabular}}

Chen et al.: LncROR and Bladder Cancer

YCSZ2015215) and self-financing of scientific research subjects of the Guangxi Health Department (Z2013472).

\section{Disclosure Statement}

The authors declare no conflict of interest

\section{Reference}

1 Jacobs BL, Lee CT, Montie JE: Bladder cancer in 2010: how far have we come? CA Cancer J Clin 2010;60:244-272.

2 Racioppi M, D'Agostino D, Totaro A, Pinto F, Sacco E, D'Addessi A, Marangi F, Palermo G, Bassi PF: Value of current chemotherapy and surgery in advanced and metastatic bladder cancer. Urol Int 2012;88:249-258.

3 Pan Y, Li C, Chen J, Zhang K, Chu X, Wang R; Chen L: The Emerging Roles of Long Noncoding RNA ROR (lincRNA-ROR) and its Possible Mechanisms in Human Cancers. Cell Physiol Biochem 2016;40:219-229.

4 Wang L, Chen Z, An L, Wang Y, Zhang Z, Guo Y, Liu C: Analysis of Long Non-Coding RNA Expression Profiles in Non-Small Cell Lung Cancer. Cell Physiol Biochem 2016;38:2389-2400.

5 Dong L, Ni J, Hu W, Yu, Li H: Upregulation of Long Non-Coding RNA PlncRNA-1 Promotes Metastasis and Induces Epithelial-Mesenchymal Transition in Hepatocellular Carcinoma. Cell Physiol Biochem 2016;38:836-846.

6 Xu B, Shao Q Xie K, Zhang Y, Dong T, Xia Y, Tang W: The Long Non-Coding RNA ENST00000537266 and ENST00000426615 Influence Papillary Thyroid Cancer Cell Proliferation and Motility. Cell Physiol Biochem 2016;38:368-378.

7 Yin DD, Zhang EB, You LH, Wang N, Wang LT, Jin FY, Zhu YN, Cao LH, Yuan QX, De W, Tang W: Downregulation of IncRNA TUG1 affects apoptosis and insulin secretion in mouse pancreatic beta cells. Cell Physiol Biochem 2015;35:1892-1904.

-8 Hua WF, Zhong Q, Xia TL, Chen Q, Zhang MY, Zhou AJ, Tu ZW, Qu C, Li MZ, Xia YF, Wang HY, Xie D, Claret FX, Song EW, Zeng MS: RBM24 suppresses cancer progression by upregulating miR-25 to target MALAT1 in nasopharyngeal carcinoma. Cell Death Dis 2016;7:e2352.

-9 Luan W, Li L, Shi Y, Bu X, Xia Y, Wang J, Djangmah HS, Liu X, You Y, Xu B: Long non-coding RNA MALAT1 acts as a competing endogenous RNA to promote malignant melanoma growth and metastasis by sponging miR-22. Oncotarget 2016;27:63901-63912.

10 Lu Y, Tan L, Shen N, Peng J, Wang C, Zhu Y, Wang X: Association of lncRNA H19 rs217727 polymorphism and cancer risk in the Chinese population: a meta-analysis. Oncotarget 2016;13:59580-59588.

11 Zhang Z, Cheng J, Wu Y, Qiu J, Sun Y, Tong X: LncRNA HOTAIR controls the expression of Rab22a by sponging miR-373 in ovarian cancer. Mol Med Rep 2016;14:2465-2472.

12 Zhu H, Lv Z, An C, Shi M, Pan W, Zhou L, Yang W, Yang M: Onco-IncRNA HOTAIR and its functional genetic variants in papillary thyroid carcinoma. Sci Rep 2016;6:31969.

-13 Loewer S, Cabili MN, Guttman M, Loh YH, Thomas K, Park IH, Garber M, Curran M, Onder T, Agarwal S, Manos PD, Datta S, Lander ES, Schlaeger TM, Daley GQ, Rinn JL: Large intergenic non-coding RNA-RoR modulates reprogramming of human induced pluripotent stem cells. Nat Genet 2010;42:1113-1117.

14 Hou P, Zhao Y, Li Z, Yao R, Ma M, Gao Y, Zhao L, Zhang Y, Huang B, Lu J: LincRNA-ROR induces epithelial-tomesenchymal transition and contributes to breast cancer tumorigenesis and metastasis. Cell Death Dis 2014;5:e1287.

15 Takahashi K, Yan IK, Kogure T, Haga H, Patel T. Extracellular vesicle-mediated transfer of long non-coding RNA ROR modulates chemosensitivity in human hepatocellular cancer. FEBS Open Bio 2014;4:458-467.

16 Zhan HX, Wang Y, Li C, Xu JW, Zhou B, Zhu JK, Han HF, Wang L, Wang YS, Hu SY: LincRNA-ROR promotes invasion, metastasis and tumor growth in pancreatic cancer through activating ZEB1 pathway. Cancer Lett 2016;374:261-271.

17 Luo G, Wang M, Wu X, Tao D, Xiao X, Wang L, Min F, Zeng F, Jiang G: Long Non-Coding RNA MEG3 Inhibits Cell Proliferation and Induces Apoptosis in Prostate Cancer. Cell Physiol Biochem 2015;37:2209-2220.

18 Tang Q Ni Z, Cheng Z, Xu J, Yu H, Yin P: Three circulating long non-coding RNAs act as biomarkers for predicting NSCLC. Cell Physiol Biochem 2015;37:1002-1009. 


\section{Cellular Physiology Cell Physiol Biochem 2017;41:2399-2410 \begin{tabular}{l|l} 
DOI: 10.1159/000475910 & $\begin{array}{l}\text { O 2017 The Author(s). Published by S. Karger AG, Basel } \\
\text { www.karger.com/cpb }\end{array}$ \\
\hline
\end{tabular}}

19 Li J, Wang X, Tang J, Jiang R, Zhang W, Ji J, Sun B: HULC and Linc00152 Act as Novel Biomarkers in Predicting Diagnosis of Hepatocellular Carcinoma. Cell Physiol Biochem 2015;37:687-696.

20 Zhou X, Ye F, Yin C, Zhuang Y, Yue G, Zhang G: The Interaction Between MiR-141 and lncRNA-H19 in Regulating Cell Proliferation and Migration in Gastric Cancer. Cell Physiol Biochem 2015;36:1440-1452.

21 Li C, Chen J, Zhang K, Feng B, Wang R, Chen L: Progress and Prospects of Long Noncoding RNAs (lncRNAs) in Hepatocellular Carcinoma. Cell Physiol Biochem 2015;36:423-434.

-22 Yang ZT, Li Z, Wang XG, Tan T, Yi F, Zhu H, Zhao JP, Zhou XF: Overexpression of Long Non-Coding RNA ZXF2 Promotes Lung Adenocarcinoma Progression Through c-Myc Pathway. Cell Physiol Biochem 2015;35:2360-2370.

23 Chen J, Lin C, Yong W, Ye Y, Huang Z: Calycosin and genistein induce apoptosis by inactivation of HOTAIR/pAkt signaling pathway in human breast cancer MCF-7 cells. Cell Physiol Biochem 2015;35:722-728.

24 Huang W, Cui X, Chen J, Feng Y, Song E, Li J, Liu Y: Long non-coding RNA NKILA inhibits migration and invasion of tongue squamous cell carcinoma cells via suppressing epithelial-mesenchymal transition. Oncotarget 2016;20:62520-62532.

-25 Li T, Xie J, Shen C, Cheng D, Shi Y, Wu Z, Deng X, Chen H, Shen B, Peng C, Li H, Zhan Q, Zhu Z: Upregulation of long noncoding RNA ZEB1-AS1 promotes tumor metastasis and predicts poor prognosis in hepatocellular carcinoma. Oncogene 2016;35:1575-1584.

26 Ma L, Tian X, Wang F, Zhang Z, Du C, Xie X, Kornmann M, Yang Y: The long noncoding RNA H19 promotes cell proliferation via E2F-1 in pancreatic ductal adenocarcinoma. Cancer Biol Ther 2016;17:1051-1061.

-27 Chen G, Wang Z, Wang D, Qiu C, Liu M, Chen X, Zhang Q, Yan G, Cui Q: LncRNADisease: a database for longnon-coding RNA-associated diseases. Nucleic Acids Res 2013;41:D983-986.

-28 Lai MC, Yang Z, Zhou L, Zhu QQ Xie HY, Zhang F, Wu LM, Chen LM, Zheng SS: Long non-coding RNA MALAT-1 overexpression predicts tumor recurrence of hepatocellular carcinoma after liver transplantation. Med Oncol 2012;29:1810-1816.

-29 Frith MC, Bailey TL, Kasukawa T, Mignone F, Kummerfeld SK, Madera M, Sunkara S, Furuno M, Bult CJ, Quackenbush J, Kai C, Kawai J, Carninci P, Hayashizaki Y, Pesole G, Mattick JS: Discrimination of nonprotein-coding transcripts from protein-coding mRNA. RNA Biol 2006;3:40-48.

30 Jia P, Cai H, Liu X, Chen J, Ma J, Wang P, Liu Y, Zheng J, Xue Y: Long non-coding RNA H19 regulates glioma angiogenesis and the biological behavior of glioma-associated endothelial cells by inhibiting microRNA29a. Cancer Lett 2016;381:359-369.

-31 Ma HW, Xie M, Sun M, Chen TY, Jin RR, Ma TS, Chen QN, Zhang EB, He XZ, De W, Zhang ZH: The pseudogene derived long noncoding RNA DUXAP8 promotes gastric cancer cell proliferation and migration via epigenetically silencing PLEKHO1 expression. Oncotarget 2016; doi:10.18632/oncotarget.11075.

32 Zhao JJ, Hao S, Wang LL, Hu CY, Zhang S, Guo LJ, Zhang G, Gao B, Jiang Y, Tian WG, Luo DL: Long noncoding RNA ANRIL promotes the invasion and metastasis of thyroid cancer cells through TGF-beta/Smad signaling pathway. Oncotarget 2016;7:57903-57918.

-33 Zhang A, Zhou N, Huang J, Liu Q, Fukuda K, Ma D, Lu Z, Bai C, Watabe K, Mo YY: The human long non-coding RNA-RoR is a p53 repressor in response to DNA damage. Cell Res 2013;23:340-350.

-34 Wang Y, Xu Z, Jiang J, Xu C, Kang J, Xiao L, Wu M, Xiong J, Guo X, Liu H: Endogenous miRNA sponge lincRNARoR regulates Oct4, Nanog, and Sox2 in human embryonic stem cell self-renewal. Dev Cell 2013;25:69-80.

-35 Chen J, Wang R, Zhang K, Chen LB: Long non-coding RNAs in non-small cell lung cancer as biomarkers and therapeutic targets. J Cell Mol Med 2014;18:2425-2436.

-36 Eades G, Wolfson B, Zhang Y, Li Q, Yao Y, Zhou Q: lincRNA-RoR and miR-145 regulate invasion in triplenegative breast cancer via targeting ARF6. Mol Cancer Res 2015;13:330-338.

-37 Zhou X, Gao Q Wang J, Zhang X, Liu K, Duan Z: Linc-RNA-RoR acts as a "sponge" against mediation of the differentiation of endometrial cancer stem cells by microRNA-145. Gynecol Oncol 2014;133:333-339.

-38 Zou G, Liu T, Guo L, Huang Y, Feng Y, Huang Q, Duan T: miR-145 modulates IncRNA-ROR and Sox2 expression to maintain human amniotic epithelial stem cell pluripotency and beta islet-like cell differentiation efficiency. Gene 2016;591:48-57.

-39 Fan J, Xing Y, Wen X, Jia R, Ni H, He J, Ding X, Pan H, Qian G, Ge S, Hoffman AR, Zhang H, Fan X: Long noncoding RNA ROR decoys gene-specific histone methylation to promote tumorigenesis. Genome Biol 2015;16:139.

-40 Lee JT: Epigenetic regulation by long noncoding RNAs. Science 2012;338:1435-1439. 